\title{
Synbiotic Supplement
}

National Cancer Institute

\section{Source}

National Cancer Institute. Synbiotic Supplement. NCI Thesaurus. Code C99230.

A nutritional supplement comprised of prebiotic and probiotic ing redients, with potential immunomodulating and gastrointestinal (Gl) flora restoring activity. Upon ingestion of the synbiotic supplement, the prebiotics and probiotics work synergistically in the Gl tract, thereby modulating the Gl flora ecosystem and may improve the functions of the intestinal barrier. In addition, synbiotics may have a beneficial effect on the immune system. 